Rev. High Pressure Sci. Technol., Vol. 7 (1998) 790 792

\title{
Structural Study of Solid Hydrogen Sulfide under High Pressure
}

\author{
H. Fujihisa, K. Aoki, H. Yamawaki, and M. Sakashita \\ National Institute of Materials and Chemical Research \\ 1-1 Higashi, Tsukuba 305, Japan
}

\begin{abstract}
An x-ray powder diffraction experiment on solid $\mathrm{H}_{2} \mathrm{~S}$ was performed with a diamond anvil cell and an image plate to determine the unknown structures of phases I' and IV up to $25 \mathrm{GPa}$ at room temperature. The structures of phases I' and IV were found to be cubic $T^{4}-P 2_{1} 3$ and tetragonal $D_{4 h}^{20}-I 4_{1} /$ acd, respectively. In phase I', the $\mathrm{H}_{2} \mathrm{~S}$ molecule had 6-fold asymmetric coordination which brought a displacement from face-center. In phase IV, the molecules were found to form spiral chains along the $c$ axis with partial loss of molecularity. The chains were linked by hydrogen bonds.

[hydrogen sulfide, hydrogen bond, phase transition, structure, pressure]
\end{abstract}

\section{Introduction}

Hydrogen sulfide $\mathrm{H}_{2} \mathrm{~S}$ forms a molecular solid in which the molecules are bound by hydrogen bonds as $\mathrm{H}_{2} \mathrm{O}$ molecules in ice. The P-T phase diagram of $\mathrm{H}_{2} \mathrm{~S}$ is very different from that of $\mathrm{H}_{2} \mathrm{O}$ ice in spite of the similarities in their molecular structures and hydrogen bonds. Many high pressure studies have been done intensively in the last five years.

Five crystalline phases are known under pressure at room temperature as follows: (1) Phase I between 0.47 to $8 \mathrm{GPa}$ has an fcc $\left(O_{h}^{5}-F_{m} \overline{3} m\right)$ structure with orientationally disordered hydrogen bonds. (2) Phase I', which has been found by an $\mathrm{x}$-ray experiment between 8 and $11 \mathrm{GPa}$ at room temperature, has a primitive cubic lattice $\left(T^{4}-P 2{ }_{1} 3\right)$ [1], but its atomic parameter has not been determined yet. (3) Phase IV is shown to exist above $11 \mathrm{GPa}$ [2], which has thin yellow color. It is stable over an extended temperature region from 30 to $300 \mathrm{~K}$ under high pressure [3]. Its structure is still unknown. (4) Phase V has been found by an x-ray experiment above 27.5 $\mathrm{GPa}$ [4] at room temperature. The color of phase $\mathrm{V}$ is black. An infrared (IR) absorption measurement [5] has shown presence of hydrogen bond system in phase V to $45 \mathrm{GPa}$. (5) Our recent IR experiment up to $100 \mathrm{GPa}$ has revealed a transition to phase VI with dissociation of $\mathrm{H}_{2} \mathrm{~S}$ molecules above $46 \mathrm{GPa}$ [6]. We have also found that $\mathrm{H}_{2} \mathrm{~S}$ becomes metallic around $96 \mathrm{GPa}$.

The optical properties have been intensively studied for solid $\mathrm{H}_{2} \mathrm{~S}$ under pressure, however, the structures have remained very uncertain. Therefore we started an x-ray powder diffraction experiment on $\mathrm{H}_{2} \mathrm{~S}$ with a diamond anvil cell (DAC) and Rietveld analysis. In the present study, we focused on phases I' and IV, and discussed their structural features.

\section{Experimental}

We prepared $\mathrm{H}_{2} \mathrm{~S}$ powder specimen as follows: Gaseous $\mathrm{H}_{2} \mathrm{~S}$ was introduced in a stainless steel bowl cooled to $77 \mathrm{~K}$ and was solidified in the bowl. Then it was ground into fine powders and was subsequently mounted on a gasketted diamond anvil cooled down to $77 \mathrm{~K}$. The DAC was immediately closed and pressurized to a few $\mathrm{GPa}$. This specimen loading procedure was carried out in a gaseous nitrogen atmosphere. We tightened screw the DAC's piston up little by little to keep the pressure sufficiently far from a melting curve on the P-T phase diagram. The finely ground powder could be neither melt nor recrystallized in this process, and was able to be retrieved at room temperature. The gasket hole size was $180 \mu \mathrm{m}$ in diameter and $120 \mu \mathrm{m}$ in thickness.

Angle dispersive powder patterns were taken with a rotatinganode $\mathrm{x}$-ray generator $(\mathrm{Mo}-\mathrm{K} \alpha)$, a pyrolytic graphite monochromator, a pin-hole collimator $(80 \mu \mathrm{m} \phi)$, and an image plate detector. The average exposure time for each pattern was 24 hours. All x-ray measurements were carried out at room temperature. A scanned image had a resolution of $50 \times 50 \mu \mathrm{m}^{2}$ per one pixel and contained $2300 \times 2300$ pixels. Smooth DebyeScherrer rings without spots were obtained as insets of Fig. 1.

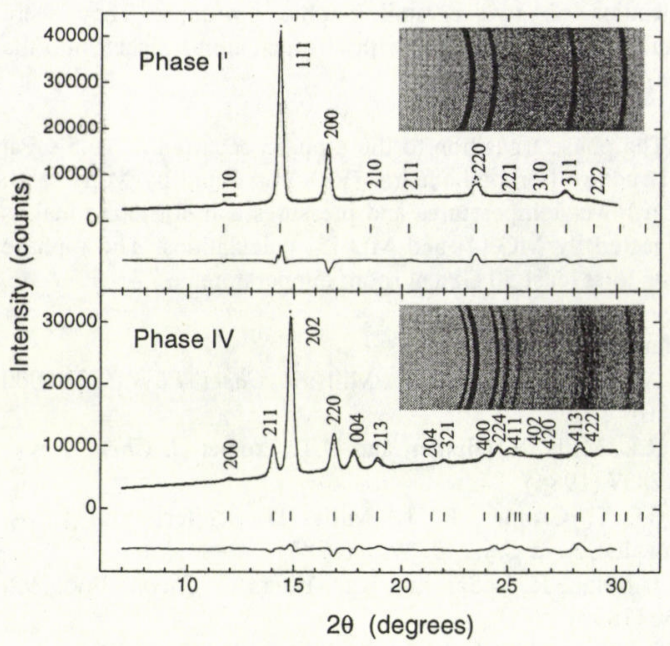

Fig. 1. Rietveld analyses of $\mathrm{H}_{2} \mathrm{~S}$ for phase I' at $9.5 \mathrm{GPa}$ (top) and for phase IV at $14.0 \mathrm{GPa}$ (bottom). Insets are parts of raw diffraction image before integration. 


\section{Analysis}

Two-dimensional diffraction images were integrated along the ring direction with an adequate $2 \theta$ step. The pattern of phase I' at $9.5 \mathrm{GPa}$ was explained well only with cubic space group $T^{4}-P 2,3$ as described in Ref. 1. The lattice and atomic parameters of sulfur were determined to be $a_{I}=4.931$ and S $4 \mathrm{a}$ : $(x=0.240 \pm 0.005, x, x)$, respectively, as the top of Fig. 1. The sulfur position in phase I' has a small displacement along $<111>$ direction when compared with an exact face-centered position as in phase I. The bottom of Fig. 1 represents a pattern of phase IV obtained at $14.0 \mathrm{GPa}$. All peaks were well indexed by tetragonal cell $a_{I V}=6.850$ and $c_{I V}=9.203$. Its space group was determined to be $D_{4 h}^{20}-I 4_{1} /$ acd. The unit cell contains sixteen $\mathrm{H}_{2} \mathrm{~S}$ molecules and corresponds to that obtained by multiplying the cubic cell of phases I and I' by $\sqrt{2} \times \sqrt{2} \times 2$. Its atomic parameter at $14.0 \mathrm{GPa}$ was determined to be $\mathrm{S} 16 \mathrm{e}:(1 / 4$, $y=0.044 \pm 0.005,1 / 8$ ) in cell choice 1 .

Figure 2 represents the pressure dependence of twelve neighboring interatomic distances of sulfur. A nearest $S$-S distance in phase $\mathrm{I}(3.544 \AA: 12$-fold at $8 \mathrm{GPa})$ split into two components (3.419 and $3.558 \AA$ : 6-fold for each) in phase I' at $9.5 \mathrm{GPa}$. They split again in phase IV, $(3.048,3.367,3.425$, 3.477 and $3.659 \AA$ : 2-, 4-, 2-, 2-, and 2-fold for each) at 14.0 $\mathrm{GPa}$, suggesting complex arrangement of $\mathrm{H}_{2} \mathrm{~S}$ molecules.

\section{Discussion}

We examine the atomic positions of hydrogen in phases I' and IV based on the positions of sulfur. A structural model of phase I' is displayed in Fig. 3. Sulfur atoms are put on the site of S $4 \mathrm{a}:(x=0.240, x, x)$ which was experimentally determined by
Rietveld analysis. We assumed that hydrogen atoms are located on the lines between the first nearest S-S distance $(3.419 \AA$ : 6 -fold) and at S-H distance of $1.3 \AA$. Then the positions of hydrogen atoms are given with sites of $\mathrm{H} 112 \mathrm{~b}:(x=0.430$, $y=0.248, z=0.058)$ and $\mathrm{H} 212 \mathrm{~b}:(0.050,0.248,0.058)$ as drawn with dark and light grays in Fig. 3, respectively. Molecules should be flipping (not ordered) to occupy these sites with an equal probability of one third to keep the first nearest S-S distance 6-fold. Such disordering in phase I' was suggested by a Raman experiment [3].

When we compare this six coordination of phase I' with the twelve coordination of low temperature phase II $\left(\mathrm{T}_{h}^{6}-\mathrm{Pa} \overline{3}\right)$ [7], a close relation between them can be found. The molecular symmetry in phase I' is $C_{3 v}-3 m$ as shown in Fig. 3 (b). Two hydrogen atoms should be paired like 1-1', 2-2', and 3-3' as labeled, since this pairing makes a reasonable molecular bond angle of 92.3 degrees which is very close to that of a free molecule. The molecular symmetry in phase II is $D_{3 d}-\overline{3} m$ as shown in Fig. 3 (c). In this case, two hydrogen atoms should be paired like 1-1', , , 6-6' to make a bond angle approximately 90 degrees. The atomic configuration in phase I' is obtained simply by losing an inversion center from that in phase II. This could occur when the molecular rotations are partially frozen by compression. This asymmetricity is responsible for the displacement of the sulfur atom along the $<111>$ direction from the face-centered position in phase I'.

The structure of phase IV has the characteristic feature of spiral chains along the $c$ axis as shown with solid lines in Fig. 4. An intrachain S-S bond, which has never been seen in lower pressure phases, would cause thin yellow color of phase IV to

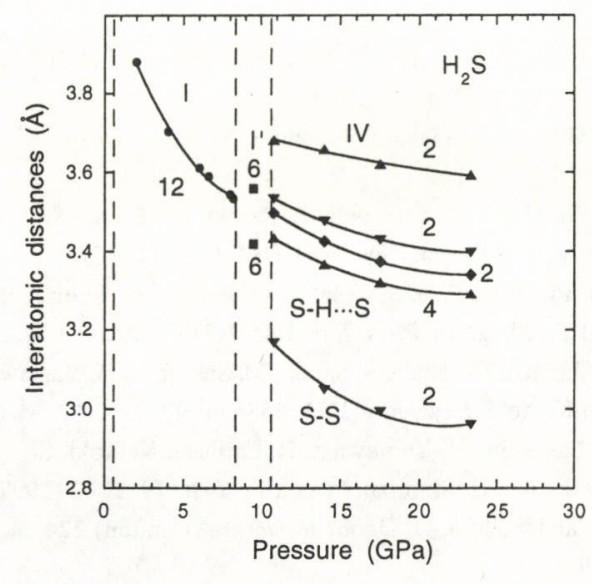

Fig. 2. Pressure dependence of neighboring S-S distances of $\mathrm{H}_{2} \mathrm{~S}$. The numbers inside mean how many equivalent distance degenerated. (a)

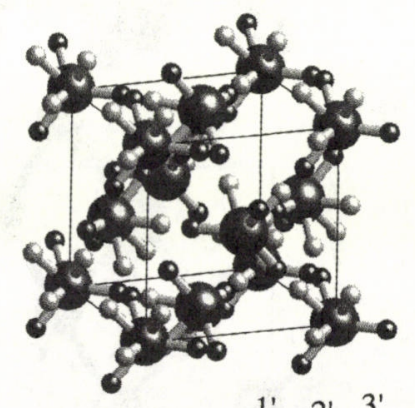

(b)

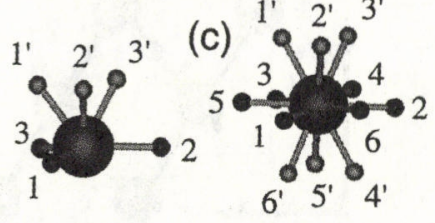

Fig. 3 (a) Structural model for phase I' of $\mathrm{H}_{2} \mathrm{~S}$.

The molecular configurations are drawn in (b) phase I' and (c) phase II. They have 6- and 12fold coordinations, respectively. 
be appeared. The hydrogen atom forms a hydrogen bond with a sulfur atom on an adjacent spiral chain as shown with dashed lines in Fig. 4. We made this model by the following considerations: The first nearest S-S distance is $3.050 \AA$ (2-fold) in phase IV at $14.0 \mathrm{GPa}$ as shown with Fig. 2. If one hydrogen atom is located on each S-S line to form a hydrogen bond, only half (16) of hydrogen atoms can exist in the unit cell. However, when we put no hydrogen atom on the first nearest $S$-S line but on the second nearest S-S line (3.367 $\AA$ : 4-fold) to form a hydrogen bond, all 32 hydrogen atoms in the unit cell can occupy a $32 \mathrm{~g}$ site of this space group. Hydrogen atoms should be located at $32 \mathrm{~g}:(x=0.412, y=0.179, z=0.288)$ in cell choice 1 to fix the molecular shape of an S-H bond length $1.3 \AA$ and a molecular bond angle 92 degrees.

The structure and bonding nature could well explain the pressure dependence of the interatomic distances of sulfur. As shown with Fig. 2, the first nearest S-S distance shortened by

(a)

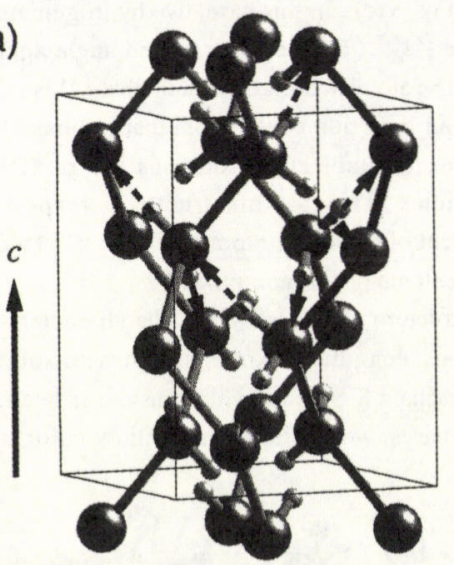

(b)

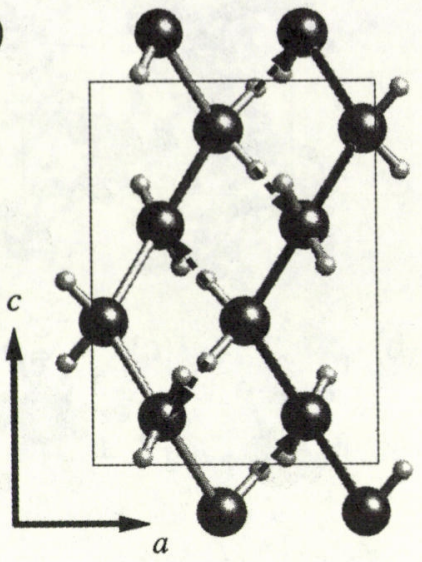

Fig. 4 Structural model for phase IV of $\mathrm{H}_{2} \mathrm{~S}$ viewed from (a) $<421>$ and (b) $<100>$ directions. Two spiral chains of four are drawn in (b). pressure effectively than the others in spite of its shorter distance. It means that the first nearest S-S bond consists of weak binding force such as van der Waals interaction (van der Waals radius of sulfur=1.85). The second nearest S-S bond consists of a hydrogen bond including hydrogen atom between sulfur atoms. Raman study [2] has shown that the symmetric S-H stretching frequency $v_{1}$ drops constantly with pressure $\left(\frac{d v_{1}}{d P}=-10.1\right.$ $\mathrm{cm}^{-1} / \mathrm{GPa}$ ) up to $23 \mathrm{GPa}$. This can be explained by our model where hydrogen bonded S-S distances in phases I and I' can connect smoothly with the second nearest S-S distance in phase IV rather than the first nearest one as shown in Fig. 2 .

The spiral chain structure has never been seen in any phases of $\mathrm{H}_{2} \mathrm{O}$ even after molecular dissociation. A variety of polymorphs in $\mathrm{H}_{2} \mathrm{~S}$ must be derived from a coexistence of hydrogen and covalent bonds. As described our previous IR study [6], optical properties of phase VI and elemental sulfur behave similarly above $46 \mathrm{GPa}$. This has been interpreted that three-dimensional S-S covalent bonds are associated, then hydrogen atoms move to interstitial positions in phase VI. Accordingly, successive transitions from IV, V, to VI would be characterized by losing hydrogen bonds and molecularity, and an increasing covalency of the S-S bonds.

The $x$-ray powder diffraction gave useful information to understand the bonding state of solid $\mathrm{H}_{2} \mathrm{~S}$. Theoretical calculations would help to understand why such spiral structure is formed, and how a hydrogen bond influences phase transitions. Further study should be done for higher pressure phases $\mathrm{V}$ and VI successively appearing along the isothermal compression at room temperature toward molecular dissociation and metallization.

\section{References}

[1] S. Endo, N. Ichimiya, K. Koto, S. Sasaki, and H. Shimizu, Phys. Rev. B 50, 5865 (1994).

[2] H. Shimizu, Y. Nakamichi, and S. Sasaki, J. Chem. Phys. 95, 2036 (1991).

[3] H. Shimizu, H. Yamaguchi, S. Sasaki, A. Honda, S. Endo, and M. Kobayashi, Phys. Rev. B51, 9391 (1995).

[4] S. Endo, A. Honda, S. Sasaki, H. Shimizu, O. Shimomura, and T. Kikegawa, Phys. Rev. B54, R717 (1996).

[5] H. Shimizu, T. Ushida, S. Sasaki, M. Sakashita, H. Yamawaki, and K. Aoki, Phys. Rev. B55, 5538 (1997).

[6] M. Sakashita, H. Yamawaki, H. Fujihisa, K. Aoki, S. Sasaki, and H. Shimizu, Phys. Rev. Lett. 79, 1082 (1997).

[7] E. Sandor and S. O. Ogunade, Nature (London) 224, 905 (1969). 